\title{
Pitiríase versicolor: isolamento e identificação das principais espécies de Malassezia
}

\author{
Pityriasis versicolor: isolation and identification of the main species
}

\author{
of Malassezia
}

\author{
Valéria Maria de Souza Framil ${ }^{1}$ \\ Márcia S. C. Melhem ${ }^{2}$ \\ Maria Walderez Szeszs ${ }^{3}$ \\ Elaine Cristina Corneta ${ }^{4}$ \\ Clarisse Zaitz ${ }^{5}$
}

Resumo: As espécies do gênero Malassezia isoladas foram: Malassezia sympodialis (16,66\%), Malassezia furfur (12,50\%), Malassezia globosa (11,45\%) e Malassezia slooffiae (2,10\%). A Malassezia sympodialis foi a espécie que predominou em nosso estudo. As espécies de Malassezia identificadas não mostraram correlação com as variantes clínicas e com a distribuição das lesões de pitiríase versicolor quanto às regiões do corpo.

Palavras-chave: Isolamento; Levedura; Malassezia; Pitiríase

\begin{abstract}
Species of the genus Malassezia isolated were: Malassezia sympodialis (16.66\%), Malassezia furfur (12.50\%), Malassezia globosa (11.45\%), and Malassezia slooffiae (2.10\%). Malassezia sympodialis predominated in the study. The species of Malassezia identified did not show correlation with clinical variants and with the distribution of pityriasis versicolor lesions in relation to areas of the body.

Keywords: Isolation; Malassezia; Pityriasis; Yeast
\end{abstract}

Gueho et al., em 1996, estudaram as características bioquímicas, morfológicas e moleculares e descreveram as sete espécies de Malassezia envolvidas na pitiríase versicolor atualmente: Malassezia furfur, $M$. sympodialis, $M$. globosa, M. obtusa, $M$. restrita, $M$. sloofiae e $M$. pachydermatis. O objetivo deste estudo foi identificar espécies de Malassezia e correlacioná-las com as variantes clínicas e a distribuição das lesões de pitiríase versicolor quanto às regiões do corpo.

O protocolo deste estudo e respectivo termo de consentimento foram aprovados pelo Comitê de Ética e Pesquisa da Irmandade da Santa Casa de Misericórdia de São Paulo. Os 102 casos de pitiríase versicolor foram confirmados por meio de diagnóstico clínico e micológico. As variantes clínicas da pitiríase versicolor foram determinadas: hipocrômicas, hipercrômicas, associação de hipocrômicas e hipercrômicas, eritematosas, foliculares e lesões circinadas. A distribuição das lesões da pitiríase versicolor quanto às regiões do corpo foi classificada em três grupos: 1) Leve: acometimento de apenas uma região do corpo (pescoço; região do tórax anterior ou posterior); 2) Moderada: acometimento de mais que uma região até três regiões do corpo (região do tórax anterior + posterior + região abdominal; região do tórax anterior + posterior + região dorsal; região do tórax anterior + braço $\mathrm{E}+$ braço $\mathrm{D}$; região do tórax anterior + posterior + pescoço; antebraço $\mathrm{E}$ ou $\mathrm{D}$;

Recebido em 10.06.2008

Aprovado pelo Conselho Consultivo e aceito para publicação em 27.11.09.

* Trabalho realizado na clínica de dermatologia da Irmandade da Santa Casa de Misericórdia

Conflito de interesse: Nenhum / Conflict of interest: None

Suporte financeiro: Nenhum / Financial funding: None

Médica segundo-assistente da clínica de dermatologia da Irmandade da Santa Casa de Misericórdia de São Paulo - São Paulo (SP), Brasil

Pesquisadora científica nível IV do Instituto Adolfo Lutz - São Paulo (SP), Brasil

Pesquisadora científica do Instituto Adolfo Lutz - São Paulo (SP), Brasil.

Aluna de mestrado em microbiologia/ICB-USP com bolsa de estudo da Capes - Coordenação de Aperfeiçoamento de Pessoal de Nível Superior - São Paulo (SP), Brasil.

Professora adjunta da clínica de dermatologia da Irmandade da Santa Casa de Misericórdia de São Paulo - São Paulo (SP), Brasil. 
TABela 1: Espécies de Malassezia isoladas em relação às variantes clínicas de pitiríase versicolor dos pacientes da clínica de dermatologia da Santa Casa de São Paulo

\begin{tabular}{|c|c|c|c|c|c|c|c|c|c|c|c|c|}
\hline \multirow{2}{*}{$\begin{array}{l}\text { ESPÉCIES } \\
\text { Malassezia } \\
\text { Variantes clínicas }\end{array}$} & \multicolumn{2}{|c|}{ M. sympodialis } & \multicolumn{2}{|c|}{ M. furfur } & \multicolumn{2}{|c|}{ M. globosa } & \multicolumn{2}{|c|}{ M. slooffiae } & \multicolumn{2}{|c|}{ Negativo } & \multicolumn{2}{|c|}{ Total } \\
\hline & $\mathrm{N}$ & $\%$ & $\mathrm{~N}$ & $\%$ & $\mathrm{~N}$ & $\%$ & $\mathrm{~N}$ & $\%$ & $\mathrm{~N}$ & $\%$ & $\mathrm{~N}$ & $\%$ \\
\hline Hipocrômicas & 15 & 15,62 & 12 & 12,50 & 10 & 10,41 & 2 & 2,10 & & 53,12 & 90 & 93,75 \\
\hline Hipercrômicas & 0 & 0 & 0 & 0 & 0 & 0 & 0 & 0 & 1 & 1,04 & 1 & 1,04 \\
\hline $\begin{array}{c}\text { Hipocrômicas } \\
+ \\
\text { hipercrômicas }\end{array}$ & 0 & 0 & 0 & 0 & 0 & 0 & 0 & 0 & 1 & 1,04 & 1 & 1,04 \\
\hline Eritematosas & 0 & 0 & 0 & 0 & 1 & 1,04 & 0 & 0 & 1 & 1,04 & 2 & 2,08 \\
\hline Lesões foliculares & 0 & 0 & 0 & 0 & 0 & 0 & 0 & 0 & 1 & 1,04 & 1 & 1,04 \\
\hline Lesões circinadas & 1 & 1,04 & 0 & 0 & 0 & 0 & 0 & 0 & 0 & 0 & 1 & 1,04 \\
\hline Total & 16 & 16,66 & 12 & 12,5 & 11 & 11,45 & 2 & 2,10 & 55 & 57,29 & 96 & 100 \\
\hline
\end{tabular}

região abdominal + região dorsal); 3) Disseminada: acometimento de mais que três regiões do corpo (pescoço + tronco + MMSS + MMII + axilas + região da virilha).

Dos 102 pacientes estudados, apenas 96 amostras biológicas foram encaminhadas para identificação das espécies de Malassezia, pois seis delas foram extraviadas. As amostras biológicas de 96 casos foram processadas na seção de micologia do Instituto Adolfo Lutz. O meio de cultura utilizado foi ágar Dixon modificado (Van Abbe, 1964), e a incubação para isolamento das colônias foi em estufa a $32{ }^{\circ} \mathrm{C}$ por período máximo de 15 dias. As provas bioquímicas utilizadas para identificação das espécies de Malassezia foram as provas da catalase, da assimilação ao teste de Tween e do teste da esculina. ${ }^{2}$ $\mathrm{O}$ método molecular para confirmação da identificação das espécies de Malassezia foi PCR-RFLP (polymerase chain reaction and restriction fragment length polymorphism analysis). ${ }^{3}$ Os dados foram armazenados em um banco de dados e comparados usando o teste do qui-quadrado e o teste de Fisher. Os valores de $\mathrm{p}<0,05$ foram considerados estatisticamente significantes.

Dos 102 pacientes com pitiríase versicolor, a forma clínica de máculas hipocrômicas foi encontrada com frequência de 94,11\%; as máculas hipercrômicas, de $0,98 \%$; a associação de máculas hipocrômicas e hipercrômicas, de 0,98\%; as máculas eritematosas, de $1,96 \%$; as lesões foliculares, de $0,98 \%$; e as lesões circinadas, de 0,98\%. A distribuição das lesões de pitiríase versicolor quanto às regiões do corpo teve o seguinte resultado: a leve ocorreu em 23,52\%; a moderada, em 56,86\%; e a disseminada, em 19,60\%. $\mathrm{Na}$ identificação das espécies do gênero Malassezia como agente etiológico da pitiríase versicolor, observou-se: a $M$. sympodialis apresentou uma frequência de 16,66\%; a $M$. furfur, de $12,50 \%$; a $M$. globosa, de 11,45\%; e a M. slooffiae, de 2,10\%. A frequência de culturas com resultados negativos foi de 57,29\%. A tabela 1 mostra as espécies de Malassezia isoladas em relação às variantes clínicas de pitiríase versicolor. As espécies de Malassezia isoladas em relação à distribuição das lesões de pitiríase versicolor quanto às regiões do corpo são observadas na tabela 2 .

A forma clínica clássica mais citada na literatura é a forma hipocrômica, e também confirmamos a sua maior frequência em nosso estudo. Estudos na literatura citam que, provavelmente, a forma hipocrômica tem sua incidência aumentada devido à exposição solar nos países tropicais. ${ }^{6,7,10}$ As demais variantes clínicas, como as lesões eritematosas, as lesões hipercrômicas, as lesões hipocrômicas e hipercrômicas, as lesões foliculares e as circinadas, também foram encontradas com baixa frequência em 
TABela 2: Espécies de Malassezia isoladas em relação à distribuição das lesões de pitiríase versicolor quanto às regiões do corpo dos pacientes da clínica de dermatologia da Santa Casa de São Paulo

\begin{tabular}{lcccccccc}
\hline $\begin{array}{l}\text { Distribuição das lesóes quanto } \\
\text { às regióes do corpo }\end{array}$ & leve & moderada & disseminada & Total \\
\hline $\begin{array}{l}\text { ESPÉCIES DE } \\
\text { Malassezia }\end{array}$ & $\mathrm{N}$ & $\%$ & $\mathrm{~N}$ & $\%$ & $\mathrm{~N}$ & $\%$ & $\mathrm{~N}$ & $\%$ \\
\hline M. sympodialis & 3 & 7,32 & 10 & 24,39 & 3 & 7,32 & 16 & 39,03 \\
M. furfur & 3 & 7,32 & 7 & 17,07 & 2 & 4,87 & 12 & 29,26 \\
M. globosa & 5 & 12,19 & 6 & 14,63 & 0 & 0 & 11 & 26,82 \\
M. slooffiae & 0 & 0 & 2 & 4,87 & 0 & 0 & 2 & 4,87 \\
\hline Total & 11 & 26,83 & 25 & 60,96 & 5 & 12,19 & 41 & 100 \\
\hline
\end{tabular}

outros estudos na literatura. ${ }^{7,10}$ A correlação entre as espécies de Malassezia e as variantes clínicas da pitiríase versicolor não foi significativa $(\mathrm{p}>0,05)$.

As espécies de Malassezia fazem parte da microbiota normal da pele. Geralmente, nos folículos pilosos de áreas seborreicas e com a presença de lípides, a levedura se transforma na sua forma parasitária como uma pseudo-hifa. ${ }^{9}$ Em nosso estudo, a grande maioria dos pacientes apresentou uma distribuição de lesões de pitiríase versicolor, quanto às regiões do corpo, na forma moderada $(56,86 \%) \mathrm{e}$ na disseminada $(19,60 \%)$, ou seja, observou-se a presença das formas parasitárias das espécies de Malassezia nas áreas ditas seborreicas e não seborreicas. A relação entre as espécies de Malassezia e a distribuição das lesões de pitiríase versicolor quanto às regiões do corpo ainda continua obscura e não foi possível comprovar uma correlação entre elas com os testes estatísticos empregados ( $p>0,05)$.

A alta frequência de culturas negativas encontrada em nosso estudo está relacionada a dois fatores principais: o melhor meio de cultura, ainda não padronizado para isolamento das espécies de
Malassezia, e o tempo entre a coleta do material clínico e seu cultivo. A recuperação das espécies de Malassezia ocorre em menos de 50\% dos casos, apesar do uso de meios de cultura específicos para otimizar o isolamento dessas leveduras. ${ }^{8}$ A predominância da $M$. sympodialis como principal agente etiológico da pitiríase versicolor em nosso estudo vem romper um conceito antigo de que apenas a $M$. furfur era o agente etiológico responsável pela pitiríase versicolor. Não foi possível demonstrar a influência das espécies de Malassezia em relação às variantes clínicas e à distribuição das lesões de pitiríase versicolor quanto às regiões do corpo.

\section{AGRADECIMENTOS}

À Capes - Coordenação de Aperfeiçoamento de Pessoal de Nível Superior pelo apoio financeiro a este trabalho. 


\section{REFERÊNCIA}

1. Guého E, Midgley G, Guillot J. The genus Malassezia with description of four new species. Antonie Van Leeuwenhoek. 1996;69:337-55.

2. Van Abbe NJ. The investigation of dandruff. $J$ Soc Cosmet Chem. 1964;15:609-30.

3. Corneta EC, Melhem MSC, Chioccola VLP, Pires MC, Keiko LO, Framil VMS, et al. Molecular identification of Malassezia species isolated from pityriasis versicolor and seborrheic dermatitis Brazilian patients. In: 16th Congress of the International Society for Human and Animal Mycology - ISHAM; 2006. Paris.

4. Forjaz MHH, Freire EL, Gama MP, Fischman O, DeLamoica-Freire EM. Pitiriase versicolor: estudo epidemiológico em voluntários da Universidade Federal de Mato Grosso (Brasil). An Bras Dermatol. 1983;58:249-52.

5. Chetty GN, Kamalam A, Thambiah AS. Pityriasis versicolor: a study of 200 cases in a tropical skin clinic. Mykosen. 1979;22:234-46.

6. Aspíroz Sancho MC, Sáenz de Santamaría MC, Moreno Borraz LA. Afecciones cutâneas relacionadas com Malassezia furfur. Rev Clin Esp. 1997;197:420-8.

7. Gupta AK, Bluhm R, Summerbell R. Pityriasis versicolor. J Eur Acad Dermatol Venereol. 2002;16:19-33.
8. Gandra RF, Melo TA, Matsumoto FE, Pires MF, Croce J, Gambale W, et al. Allergenic evaluation of Malassezia furfur crude extracts. Mycopathologia. 2002;155:183-9.

9. Zaitz C, Ruiz LRB, Souza VM. Dermatoses associadas às leveduras do gênero Malassezia. An Bras Dermatol. 2000;75:129-42.

10. Crespo Erchiga V, Delgado Florencio V. Malassezia species in skin diseases. Curr Opin Infect Dis. 2002; 15:133-42

ENDEREÇO PARA CORRESPONDÊNCIA / MAILING ADDRESS: Valéria Maria de Souza Framil

Rua Sete de Abril, 296, $1^{\circ}$ andar / CJ: 11, 01044000 República São Paulo, SP. Tel./Fax: 11996619601132578978. E-mail: souza.valeria@terra.com.br

Como citar este artigo/How to cite this article: Framil VMS, Melhem MSC, Szeszs MW, Corneta EC, Zaitz C. Pitiríase versicolor: isolamento e identificação das principais espécies de Malassezia. An Bras Dermatol. 2010;85(1):111-4. 